\title{
Research and Design of Enterprise Network Data Center Based on Cloud Computing
}

\author{
ZHANG Da-wei ${ }^{a}$, YANG Yong, LI Hai-yan \\ Modern Educational Technology Center, Kunming University, Yunnan Kunming 650214, China \\ aEmail: Dwzhangkm202@163.com
}

Keywords: Cloud Computing; Enterprise Network Data; intelligence firefly algorithm

\begin{abstract}
On the basis of cloud model, proposed the intelligence firefly algorithm, analyzes the position, strengths and weakness in objective function's quantization individuals of solving problems. We propose the applications of improved firefly algorithm in cloud computing task scheduling. Starts with the need of resources allocation in cloud computing environment, be similar with intelligence firefly algorithm in resources allocation, this text improves the update of fluoresce in based on researches of intelligence firefly algorithm, makes it better adapt to the resources allocation in cloud computing, through simulation experiment, the improvement of this algorithm has certain effect, and sets a theory basis for further improve the system's resources allocation effect under cloud computing model.
\end{abstract}

\section{Introduction}

Cloud computing is a now wildly used architecture hot, it's product of the development of grid computing, distributed computing, network storage and parallel processing [1]. It shows that the user's applications can operate without personal computer but the server cluster in the Internet. There are three basic forms of cloud computing services including: Infrastructure as a Service (IAAS), Platform as a Service (PAAS) and Software as a Service (SAAS) [2]. In cloud computing, the allocation of resources is a very important issue, the unsatisfactory allocation of resources can easily led the cloud's servers crashed and other servers in idle. So in cloud environment, the problem mostly need to solve is the ways to control any server's resources allocation and use condition by the information communication of local and in the Internet to make better use of the resources. Literature [3] made researches of the resources allocation conditions in different environment. Literature [4] proposed the resources allocation mechanism of self-management, self-adjustment and self-protection. Literature [5] proposed a resources allocation system applies to extensive distributed system, which efficiently increased the system's service quality under cloud computing.

\section{Task scheduling in Enterprise Network Data Center Based on Cloud Computing}

The cloud model is a transformation model uses linguistic values to express the uncertainty between a certain conception and its quantification expression, it fully combines fuzziness and randomness and forms the mapping between qualitative and quantification, shows in figure 1 .

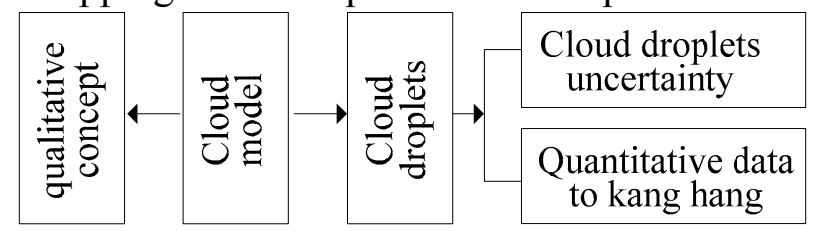

Figure1. Transformation schematic of cloud mode's qualitative concept and quantitative data

Sets $U$ is discourse domain expressed by accurate numerical value, $A$ is corresponding qualitative concept in U. If quantitative value $x \in u$ and $\mathrm{x}$ is a random implementation with likely normal distribution of qualitative concept $A$ in discourse domain $U$, the certainty degree $A(x) \in[0,1]$ of $\mathrm{x}$ to $\mathrm{A}$ is also a random number with likely normal distribution, then data array $\left(x, A\left(x_{i}\right)\right)$ is called as cloud drop, the whole element ${ }^{x_{i}}(i=1,2 \ldots n)$ in discourse domain $\mathrm{U}$ and its 
certainty degree $A\left(x_{i}\right)$ for $\mathrm{A}, i, e$, $\mathrm{n}$ data array $\left(x, A\left(x_{i}\right)\right)$, forms the cloud model with $\mathrm{n}$ cloud drop, calls $\mathrm{x}$ distribution in discourse domain $\mathrm{U}$ as cloud distribution. The number characteristics of cloud model are expressed as expectation (Ex), entropy (En) and excess entropy (He). Among them, expectation (Ex) refers to the central value of discourse domain $U$, is the center of qualitative concept, reflects the cloud focus of the whole cloud drop swarm; entropy (En) refers to the range which can be received by fuzzy concept, $E n \succ 0$; excess entropy $(\mathrm{He})$ is a uncertain measurement of entropy, i,e, the excess entropy is the entropy's entropy, He $>0$. The excess entropy reflects the degree of reach an agreement of cloud drop of representation qualitative concept or the concentration degree of cloud drop's representation qualitative concept; the bigger excess entropy is, the qualitative concept has worse common sense or the qualitative concept is worse decentralization.

The intelligence firefly algorithm, proposed by KRISHNANAD, etc. in 2005, it's a new intelligence swarm optimal algorithm, this algorithm is wildly used in producing and scheduling, its simulated the search and optimize process to the firefly's attraction and migration, measured the advantages and disadvantages of the individual's position by solving the objective function. In this algorithm, each intelligence firefly distributes in the declaration space of objective function, this intelligence firefly has its field of view and carries fluorescent powder, the brightness of fireflies is related to its position and the fitness value of objective function, the brighter position shows the firefly there has pretty objective value and it can attract more fireflies to move towards this direction, as each firefly has its own range of view, the range can be affected by the neighbor fireflies, when the number of firefly becomes fewer, the range of view is larger and attracts more fireflies. When the fireflies are more, the range of view becomes smaller. But at last the position which most of the fireflies in is the optimal solution position.

Suppose the firefly swarm id N, the $i$ firefly's position ${ }^{\left(x_{i}, y_{i}\right)}$ matches the objective function $f\left(\left(x_{i}, y_{i}\right)\right)$ and the firefly's fluoresce in value is ${ }^{T_{i}}$, the updating formula of each firefly's range of view is:

$$
f_{k}^{i}(u+1)=\min \left\{f_{t}, \max \left\{0, f_{k}^{i}(u)+\beta\left(t_{u}-\left|t_{u}(u)\right|\right)\right\}\right\}
$$

Among them, $f_{k}^{i}(u+1)$ is the COOLEYE of $i$ firefly in $u+1$ range of view, $t_{u}$ is the threshold value of the neighbor firefly's number. $\beta$ is control constant, $t_{u}(u)$ is the number of firefly with high fluoresce in range of view. There, the formula of $t_{u}(u)$ is as following:

$$
t_{i}(u)=\left\{j:\left\|y_{j}(u)-y_{i}(u)\right\| \prec f_{k}^{i} l_{i}(t) \prec l_{j}(u)\right\}
$$

There, $y_{j}(u)$ is the position of $\mathrm{j}$ firefly in $\mathrm{t}$ generation, $l_{j}(u)$ is the ${ }^{j}$ firefly's fluoresce in value in $t$ generation, the view of range between neighbor firefly is in $f_{k}^{i}$.

The firefly neighbor's selecting probability is:

$$
f_{i j}(u)=\frac{l_{i}(u)-l_{j}(u)}{\sum_{k \in n_{i}(u)} l_{k}(u)-l_{i}(u)}
$$

The position updating formula of firefly:

$$
f_{i}(u)=f_{i}(u-1)+s \frac{f_{j}(u-1)-f_{i}(u-1)}{\left\|f_{j}(u-1)-f_{i}(u-1)\right\|}
$$

Fluoresce in value's formula:

$$
f_{i}(u+1)=(1-t) l_{i}(u)+\gamma k\left(c_{i}(u+1)\right)
$$

In formula (5), $\gamma$ is a parameter to measure the function value, $k\left(c_{i}(u+1)\right)$ is the fitness value of the function.

In nature, supposes firefly $i$ enters into the view range of ${ }^{j}$, and the fluorescence in value is bigger than itself. Firefly ${ }^{i}$ chooses firefly $\mathrm{j}$ according to probability $t_{i j}(u)$, after choosing, the 
position of firefly ${ }^{i}$ and the fluorescence in is updating, calculates the objective function value of this position.

\section{Test and results}

In order to prove the performance of this algorithm, tests in two aspects, one is the performance of algorithm, the other is the task scheduling in cloud computing.

The study makes comparison test using three benchmark functions in literature and to test the algorithm's efficiency and performance [5]. Using of MATLAB in Windows.

Sphere function

$$
f(x)=\sum_{i=1}^{m} x_{i}^{2}-100 \leq x_{i} \leq 100
$$

This is a continuous, un-modal convex function, the minimum point of the global function is zero, ${ }^{i, e, x_{i}}=0(i=1,2, \ldots n)$, there are on interaction among variables.

Goldstein-Price function

$$
\begin{aligned}
& f(x)=\left[1+\left(x_{1}+x_{2}+1\right)^{2}+\left(18-13 x_{1}+2 x_{1}^{2}+5 x_{1} x_{2}+2 x_{2}^{2}\right)\right] \\
& {\left[30+\left(2 x_{1}-3 x_{2}\right)^{2}\left(19-33 x_{1}+12 x_{1}^{2}+27 x_{2}^{2}\right)\right]-3 \leq 2, i=\{1,2\}}
\end{aligned}
$$

This is a multiple hump function, the minimum point of the whole function is $3, i, e, x_{i}=3(i=1,2, \ldots n)$, there are on interaction among variables.

Ackely function

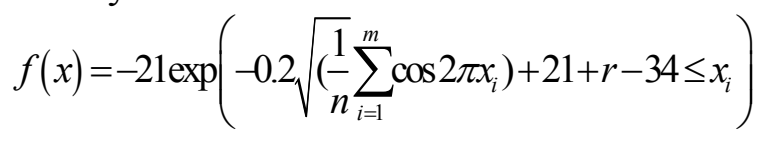

This is a multiple hump function with many local minimum points, the minimum point of the whole function is $0,{ }^{i, e, x_{i}}=0(i=1,2, \ldots n)$, there are on interaction among variables.

In the setting process of algorithm parameter, the scale of initial firefly is 500, iteration time is 200 , parameter of fluoresce in is $p=0.6$, parameter of function is $\gamma=0.4$, initial fluoresce in is $l_{0}=10$.

Respectively tests 10 times of three functions and gets the best solution, worst solution and the average value. According to make compare with basic firefly algorithm, the results is shown in table 2. It is the convergence curve comparison between the algorithm in this text and intelligence firefly algorithm in three functions. The comparison curves of different algorithms were showed as figure 4-6.

The improved firefly algorithm is obviously better than intelligence firefly algorithm no matter in the best solution, the worst solution or the average. When basis intelligence firefly algorithm has certain iterations, it's easily fall into local optimum. This text efficiently limits the probability of

\begin{tabular}{|c|c|c|c|c|}
\hline Function & Algorithm & Best solution & Worst solution & Average \\
\hline \multirow{2}{*}{ Sphere function } & $\begin{array}{l}\text { intelligence } \\
\text { algorithm }\end{array}$ & 0.0212501 & 0.0298742 & 0.0255600 \\
\hline & algorithm in this text & 0.0019610 & 0.0105412 & 0.0062476 \\
\hline \multirow{2}{*}{$\begin{array}{l}\text { Goldstein-Price } \\
\text { function }\end{array}$} & $\begin{array}{ll}\text { intelligence } & \text { firefly } \\
\text { algorithm } & \end{array}$ & 3.0005126 & 3.0014521 & 3.0009822 \\
\hline & algorithm in this text & 3.0000003 & 3.0000301 & 3.0000112 \\
\hline \multirow{2}{*}{$\begin{array}{l}\text { ACKELY } \\
\text { function }\end{array}$} & $\begin{array}{ll}\text { intelligence } & \text { firefly } \\
\text { algorithm } & \end{array}$ & 3.4589131 & 3.6521469 & 3.5565298 \\
\hline & algorithm in this text & 3.2456321 & 3.3562153 & 3.3109179 \\
\hline
\end{tabular}
felling into local optimum according to improve the fluoresce in of firefly, therefore reaches the optimum time of objective function.

Table 1.Sub-tasks and resources table 


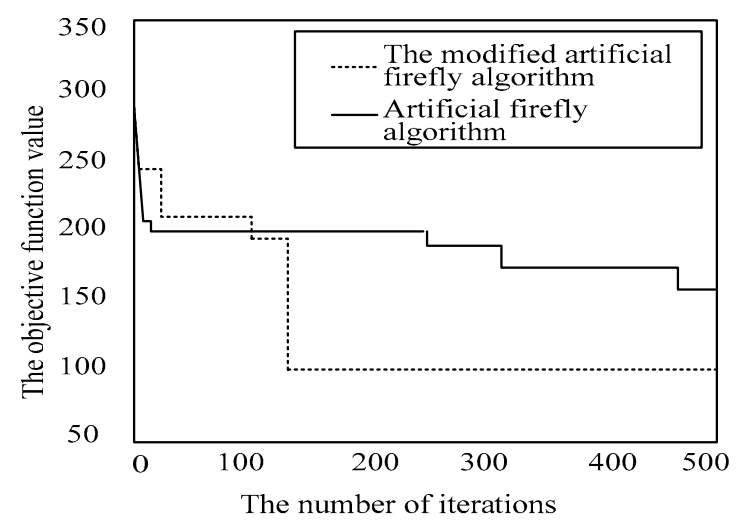

Figure2. Comparison of Sphere function's convergence curves

\section{References}

[1]Peng J.C., Gu L.Z., Su J.B., The Hand Tracking for Humanoid Robot Using Camshift Algorithm and Kalman Filter. Journal of Shanghai Jiaotong University, Vol. 40, No. 7, pp. 1161-1165, 2006.

[2]Y. Geng, J. He, H. Deng and K. Pahlavan, Modeling the Effect of Human Body on TOA Ranging for Indoor Human Tracking with Wrist Mounted Sensor, 16th International Symposium on Wireless Personal Multimedia Communications (WPMC), Atlantic City, NJ, Jun. 2013.

[3]Horst F. Wedde, Muddassar Farooq. A comprehensive review of nature inspired routing algorithms for fixed telecommunication networks, Journal of Systems Architecture, Vol. 52, No. 8-9, pp. 461-484, August- September 2006

[4]Y. Geng, J. He, H. Deng and K. Pahlavan, Modeling the Effect of Human Body on TOA Ranging for Indoor Human Tracking with Wrist Mounted Sensor, 16th International Symposium on Wireless Personal Multimedia Communications (WPMC), Atlantic City, NJ, Jun. 2013.

[5]Y. Geng, J. He, K. Pahlavan, Modeling the Effect of Human Body on TOA Based Indoor Human Tracking[J], International Journal of Wireless Information Networks 20(4), 306-317 